\title{
A Multiplexing Filtering Antenna
}

\author{
Chun Xu Mao, Member, IEEE, Long Zhang, Member, IEEE, Mohsen Khalily, Senior Member, IEEE, \\ Yue Gao, Senior Member, IEEE, and Pei Xiao, Senior Member, IEEE
}

\begin{abstract}
In this paper, a compact, highly integrated multiplexing filtering antenna operating at 4.7/5.2/6.0/6.6 GHz is proposed for the first time. Different from traditional antennas, the proposed antenna has one shared radiator but four ports working in different frequency bands and thus, it can simultaneously support four different transmission channels. The proposed multiplexing antenna is composed of a patch with a U-shaped slot, two substrate integrated waveguide (SIW) cavities, and four resonator-based frequency-selective paths. The resonator-based paths can not only enhance the inter-channel isolations but also improve the impedance bandwidth. The design principles and the methods of controlling the four operating bands are studied. Measurement results agree reasonably well with the simulations, showing four channels from 4.5 to $4.8 \mathrm{GHz}, 5.1$ to $5.3 \mathrm{GHz}, 5.85$ to $6.3 \mathrm{GHz}$, and 6.4 to $6.6 \mathrm{GHz}$, respectively. The antenna also exhibits a high isolation of over $25 \mathrm{~dB}$ between the channels. In addition, the proposed antenna has a consistent broadside radiation pattern and polarization in the four bands, manifesting the proposed multiplexing filtering antenna can be a promising candidate for multi-service wireless communication systems.
\end{abstract}

Index Terms-Antenna, filtering antenna, duplexing, multiplexing.

\section{INTRODUCTION}

With the rapid development of wireless communication technologies, high-integration radio frequency (RF) frontends with multiple functionalities are highly desired for system miniaturization. Recently developed filtering antenna, as a co-designed filter-antenna can not only reduce insertion loss due to the mismatch at the interfaces but also improve the frequency responses [1]-[8]. To date, most of the reported filtering antennas focus on improving the bandwidths [1]-[3], gains [4]-[5], frequency selectivity [6]-[7], and out-of-band suppression [8]. Although frequency responses of the filtering antennas are significantly improved as compared with conventional antennas, they are restricted to the single-port component.

In multi-band or multi-standard communication systems (e.g., 3G, $4 \mathrm{G}$, and 5G), a multi-channel antenna with different operating bands but consistent polarization at each port can significantly reduce not only the interference between different antennas/bands for different services but also the footprint of the antenna. One key requirement of duplexing antennas is to obtain high isolation between the ports/channels, which is very challenging, especially when the operating channels/bands are close to each other. Other challenges include consistent polarization, broad bandwidths, and improved frequency selectivity at different frequency bands. Recently, dual-port duplexing antennas with integrated duplexing and filtering functions were reported for frequency division duplexing (FDD) wireless applications [9]-[20]. In [9]-[14], duplexing filtering antennas/arrays

Manuscript submitted on July 10, 2020; This work was supported by the U.K. Engineering and Physical Sciences Research Council (EPSRC) under Grant EP/P008402/2. (Corresponding author: Chunxu Mao, Long Zhang).

Chunxu Mao, Mohsen Khalily, Yue Gao, and Pei Xiao are with Institute for Communication Systems, 5G Innovation Center (5GIC), University of Surrey, Guildford GU2 7XH, U.K. (e-mail: c.mao@ surrey.ac.uk).

Long Zhang is with the College of Electronics and Information Engineering, Shenzhen University, Shenzhen 518060, China. with consistent linear polarization were reported. Good filtering performance with high inter-channel isolations over $30 \mathrm{~dB}$ was achieved. Duplexing filtering antennas with circularly polarized (CP) [15]-[16] and dual-polarized [17] properties were also investigated. To date, most reported multi-channel antennas focused on two ports duplexing operation and it is essential to increase the ports/channels by using one single radiator while maintaining a consistent polarization and high isolation. In [19], an antenna-triplexer was proposed. However, the antenna has different polarizations in the three bands/channels and no integrated filtering function was achieved.

In this paper, a four-channel multiplexing antenna with integrated filtering functionality is proposed for the first time for FDD wireless communication systems. The antenna features a single slotted patch as the radiating element shared by four separate ports, each of which supports a separated frequency band/channel. The antenna also exhibits consistent radiation pattern and polarization in the four bands/channels. To improve the bandwidth and filtering performance while enhancing the isolations between them, four resonator-based filtering circuits with different resonances are integrated at the four ports. Thanks to the shared aperture by the four operating channels, the multiplexing filtering antenna exhibits a very compact size as compared with the separated multiple-antenna scheme that four individual patches with different resonant frequencies are required. Moreover, the antenna is capable of suppressing the out-of-band interference due to the integrated resonant structures.

The remainder of this paper is organized as follows. Section II illustrates the proposed filtering multiplexing antenna and its design approaches. Section III presents the experimental and the measured results of the antenna, followed by concluding remarks in Section IV.

\section{Multiplexing Filtering Antenna Design}

In this section, the proposed multiplexing filtering antenna operating at four frequency bands $(4.7 / 5.2 / 6.0 / 6.6 \mathrm{GHz})$ is first illustrated. Then, the approaches to implementation and design considerations are explained. The guidelines for performing the design are provided in the end.

\section{A. Antenna Configurations}

Fig. 1(a) shows the structural configuration of the proposed multiplexing filtering antenna. The antenna consists of two dielectric substrates and a thin foam between them. Different from traditional antennas, this multiplexing antenna has four ports (P1, P2, P3, and P4), corresponding to four operating channels/frequency bands. In all these channels, the antenna has a consistent linear polarization along the $\mathrm{x}$-axis direction. The rectangular patch with a U-shared slot is printed on the top layer of the upper substrate. The U-shaped slot in the patch is used to introduce an additional resonant mode for realizing the dual-band operation, which will be detailed in the Part-C of this section. In the lower substrate, two cavities based on substrate integrated waveguide (SIW) with two U-slots slit on the top layer are embedded, as illustrated in Fig. 1(b). The SIW cavity is formed by connecting the top layer of the middle substrate and the bottom layer of the lower substrate using metallic via holes. The two U-slots in the cavities are used to excite the patch and tune the resonant frequencies 


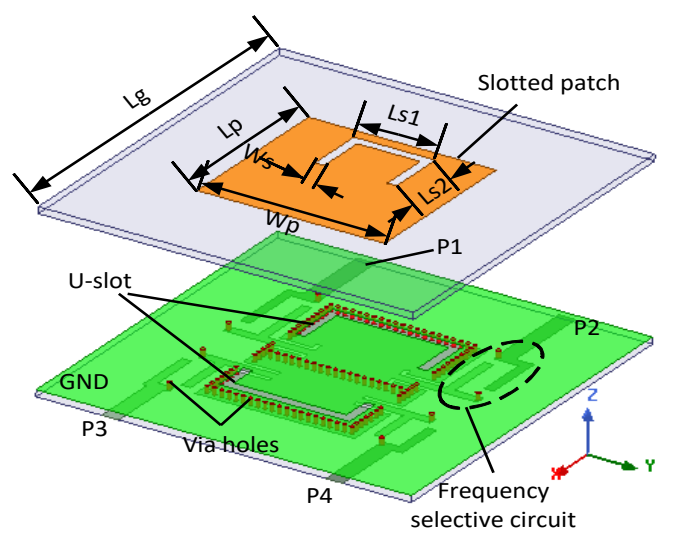

(a)

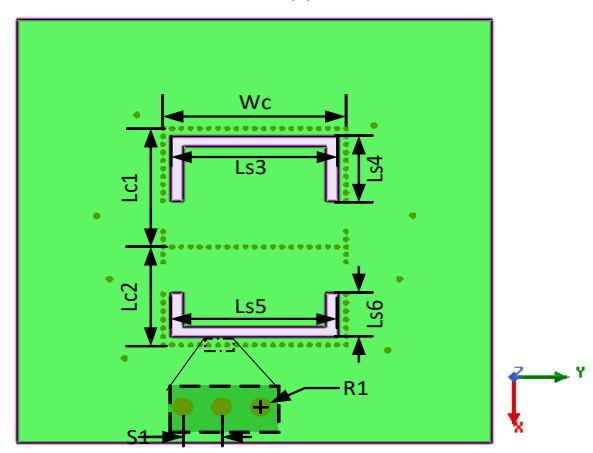

(b)

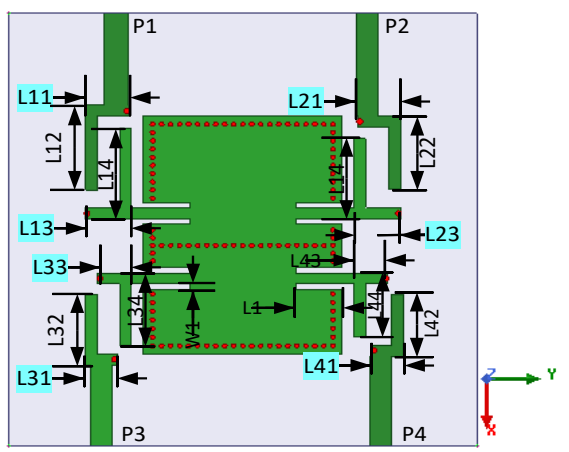

(c)

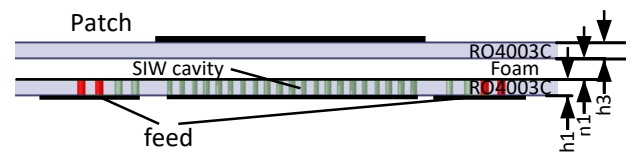

(d)

Fig. 1. configuration of the proposed multiplexing antenna, (a) exploded view, (b), ground plane with etched U-slot lines, (c) feeding networks on the bottom layer, and (d) side view.

of the cavities, which will be detailed in Part-D. In the bottom layer of the lower substrate, four resonator-based feeding networks, corresponding to the four channels/bands are conceived as the frequency selectivity circuits, which will be discussed in Part-E of this section. Fig. 1(d) shows the side view of the antenna. Rogers 4003C with a relative permittivity of 3.55 and a loss tangent of 0.0027 is used as the substrate. The thickness of the foam between the two substrates is $1 \mathrm{~mm}$. Although the proposed antenna can simultaneously support four different channels/operating bands, it has a compact size of $0.62 \lambda$ $\times 0.62 \lambda @ 4.7 \mathrm{GHz}$ due to the employed integrated design approaches and the shared radiating element. The design and optimization of the antenna was carried out using the High Frequency Structural Simulator (HFSS) and the optimal dimensional values are listed in the Table-I.
Table I DIMENSIONAL VALUES OF THE PROPOSED ANTENNA (Unit: mm)

\begin{tabular}{cccccc}
\hline \hline$L g$ & $L p$ & $L s 1$ & $L s 2$ & $W p$ & $W c$ \\
40 & 18.5 & 8.7 & 6.5 & 20 & 15.4 \\
\hline$L c 1$ & $L c 2$ & $L s 3$ & $L s 4$ & $L s 5$ & $L s 6$ \\
11.6 & 9.3 & 14 & 6.2 & 14 & 4.2 \\
\hline$L 1$ & $W 1$ & $L 11$ & $L 12$ & $L 13$ & $L 14$ \\
4 & 0.5 & 8.3 & 3.7 & 3.5 & 8.5 \\
\hline$L 21$ & $L 22$ & $L 23$ & $L 24$ & $L 31$ & $L 32$ \\
3.8 & 6.8 & 4 & 6.6 & 2.7 & 6.5 \\
\hline$L 33$ & $L 34$ & $L 41$ & $L 42$ & $L 43$ & $L 44$ \\
2.8 & 6.4 & 2.8 & 5.8 & 2.8 & 5.8 \\
\hline$R 1$ & $S 1$ & $h 1$ & $h 2$ & $h 3$ & $W s$ \\
0.2 & 0.8 & 0.8 & 1 & 0.8 & 1 \\
\hline \hline
\end{tabular}

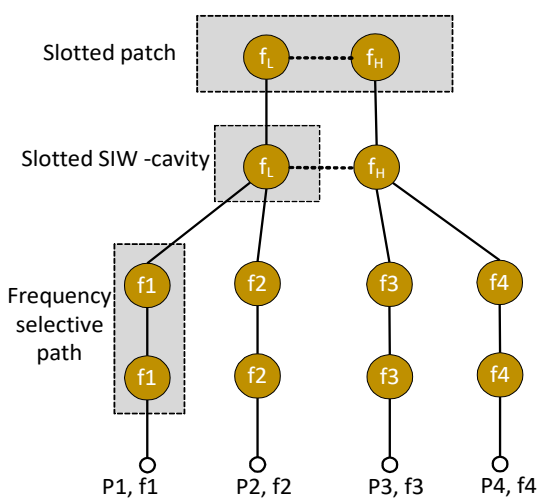

Fig. 2. Resonator-based topology of the proposed multiplexing filtering antenna.

\section{B. Operating Principles}

In order to illustrate the operating principles of the proposed multiplexing filtering antenna, a resonator-based topology is presented, as shown in Fig. 2. The circles represent the resonant elements and the lines represent the mutual couplings between them. It should be noted that the slotted patch can generate two resonant frequencies, and therefore, is modelled by two resonators, denoted as $f_{L}$ and $f_{H}$, respectively. The two slotted SIW cavities are modelled by two resonators, which have the same resonant frequency as the slotted patch. By using the strong mutual couplings between the patch and the cavities, each pair of coupled resonators can generate two different resonant frequencies. Thus, totally four resonant frequencies can be achieved by the coupled patch and cavities. Then, four frequency selective paths are used to select the signals at the four channels/bands and output from four ports, respectively. Each frequency selective path is composed of two resonators with a given resonant frequency to enhance the isolation between different channels. Thus, the $3^{\text {rd }}$-order filtering responses for each channel can be formed.

Fig. 3 illustrates the simulated current distribution on the bottom layer of the antenna when each port is excited, respectively. As observed from Fig. 3(a), when Port-1 is excited at $4.7 \mathrm{GHz}$, very strong current intensity can be observed on the corresponding feed line and the frequency selective path. The current intensity is significantly decreased at the other ports, showing a weak mutual coupling between the ports/channels. The same behaviour can also be observed when the other three ports are excited, as shown in Figs. 3(b)-(d), respectively.

Fig. 4 shows the simulated current vector distribution on the patch. As observed, the current mainly flows along the edges of the patch at the two lower operating bands but flows along the U-shaped slot at the two higher bands, indicating different lengths of the current paths. We can also notice that the total current is along with the $\mathrm{x}$-axis direction and the current component in the $y$-axis direction is cancelled out, 


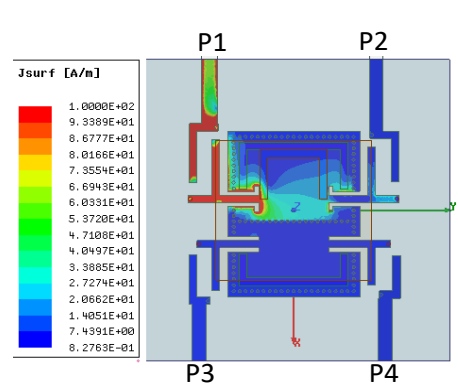

(a)

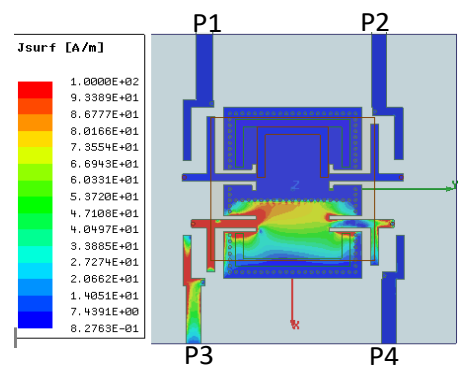

(c)

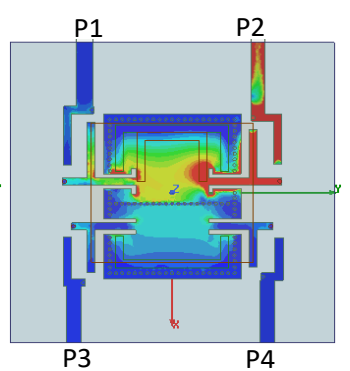

(b)

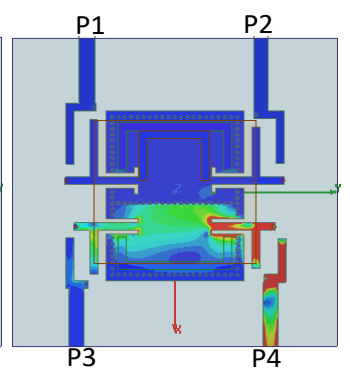

(d)
Fig. 3. Simulated current distribution on the bottom layer, (a) Port-1 is excited, $4.7 \mathrm{GHz}$, (b) Port-2 is excited, $5.2 \mathrm{GHz}$, (c) Port-3 is excited, 6.0 $\mathrm{GHz}$, (d) Port-4 is excited, 6.6 GHz.

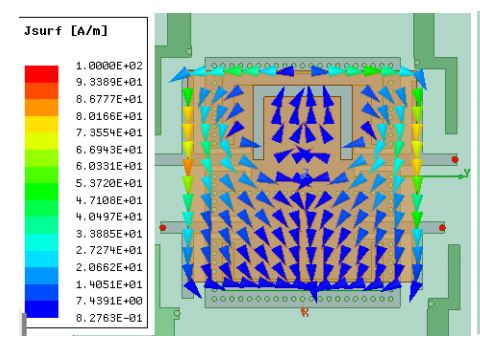

(a)

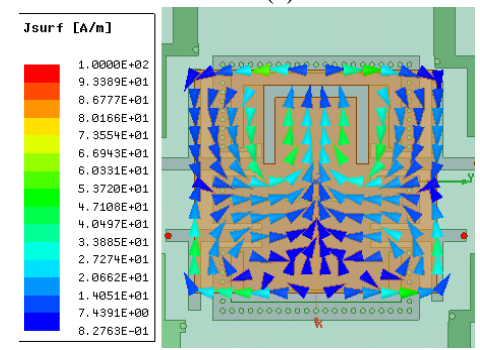

(c)

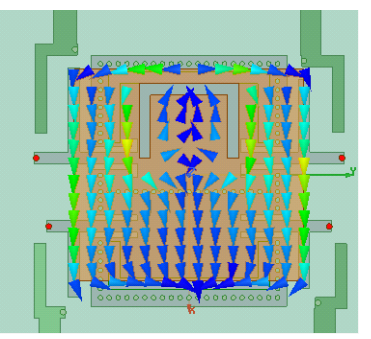

(b)

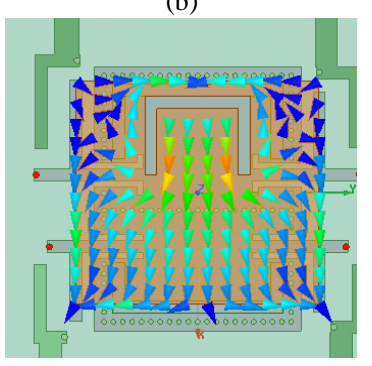

(d)
Fig. 4. Simulated current distribution on the patch, (a) Port-1 is excited, 4.7 $\mathrm{GHz}$, (b) Port-2 is excited, $5.2 \mathrm{GHz}$, (c) Port-3 is excited, $6.0 \mathrm{GHz}$, (d) Port-4 is excited, $6.6 \mathrm{GHz}$.

demonstrating the antenna has the identical linear polarization in the $\mathrm{x}$-axis direction. Due to the current paths at the four channels are varied and restricted in some specific areas of the patch, which is different from the current distribution of a conventional patch, the radiation efficiency is somehow lower than a conventional antenna.

\section{Dual-Resonant Slotted Patch}

According to the topology, dual-resonant characteristics is required for the radiating element to achieve the four-channel multiplexing operation. In order to meet this requirement, a U-shaped slot is slit in the patch to generate additional resonance [21]. To simplify the analysis of the slotted patch, the patch part in Fig. 1 is fed by a straight slot in the ground plane and replotted in Fig. 5(a). Fig. 5(b)-(c) present the simulated current distribution of the patch at the two resonant

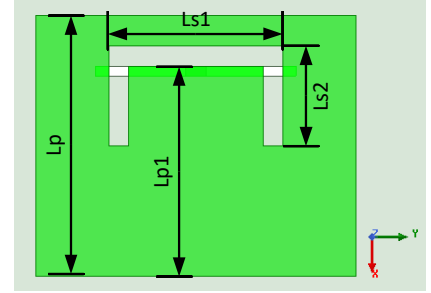

(a)

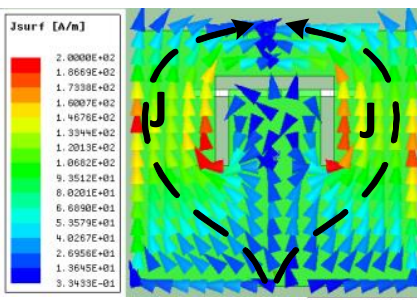

(b)

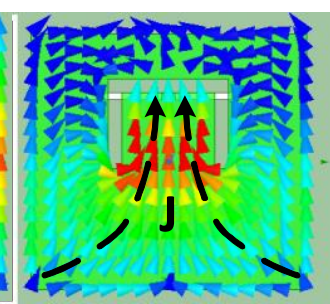

(c)
Fig. 5. Slotted patch: (a) configuration, (b) simulated current distribution at $5 \mathrm{GHz}$, and (c) simulated current distribution at $6.6 \mathrm{GHz}$.

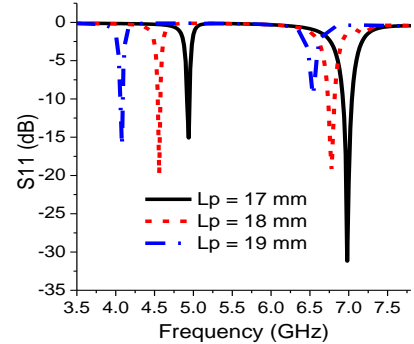

(a)

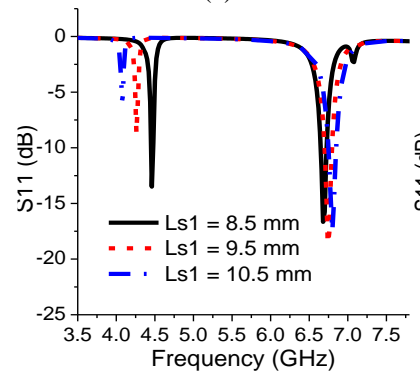

(c)

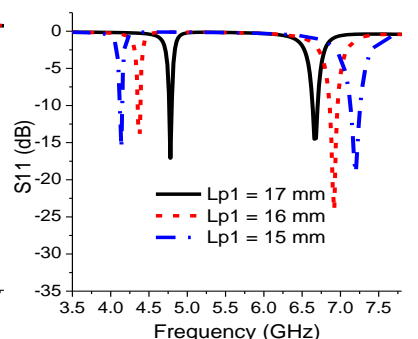

(b)

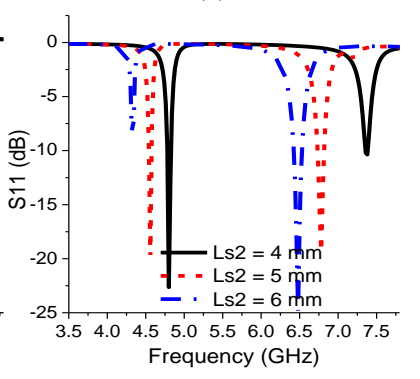

(d)

Fig. 6. Simulated $\mathrm{S}_{11}$ of the slotted patch vary with (a) $L p$, (b) $L p 1$, (c) $L s 1$, and (d) $L s 2$.

frequencies (5 and $6.6 \mathrm{GHz}$ ), respectively. One can see that when the antenna resonates at the lower mode $(5 \mathrm{GHz})$, the current flows along the two sides of the U-shaped slot, forming a bent and lengthening current distribution path. In contrast, the current flows from one side of the patch to the inner part of the U-shaped slot at the high mode (6.6 $\mathrm{GHz}$ ), resulting in a shortened path. For both cases, the current distribution is along the $\mathrm{x}$-axis direction, which ensures a consistent polarization at the two bands.

To illustrate the methods of how to control the two resonances, a parametric study is carried out and presented in Fig. 6. Viewing the current paths in Fig. 5, it is obvious that the length of the patch $(L p)$ and location of the U-slot ( $L p l)$ determine the lengths of the current paths at both bands, respectively. As a result, the resonant frequencies can be changed accordingly. It should be noted that the two modes move towards the same direction when $L p$ is varied, but they move towards opposite directions when $L p 1$ is changed. This provides a feasible approach to achieve two resonances with an arbitrary ratio. In addition, the two resonances can be tuned by adjusting the dimensions of the U-slot. As can be observed from Figs. 6(c)-(d), the value of Ls1 is sensitive to the lower resonance but insensitive to the higher 


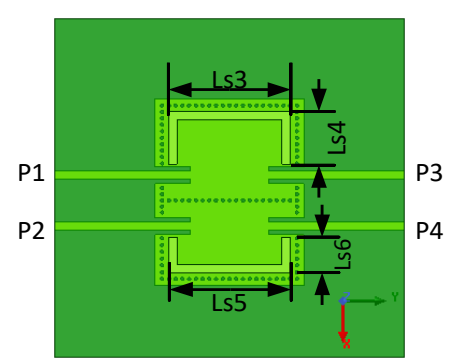

(a)

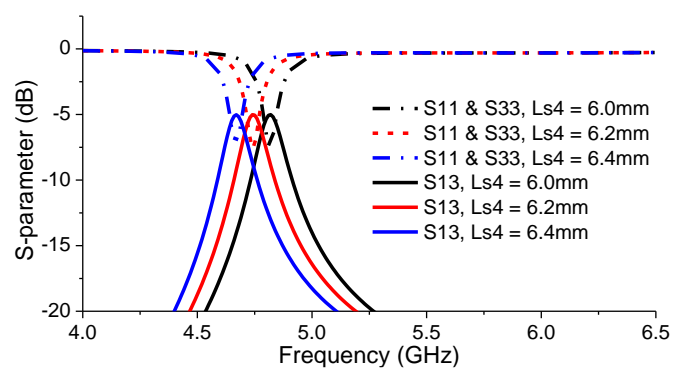

(b)

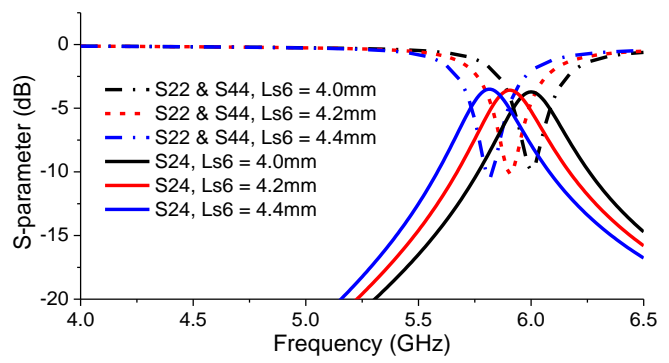

(c)

Fig. 7. Slotted SIW cavities: (a) configuration, (b) simulated S-parameters with different $L s 4$, (c) simulated S-parameters with different Ls6.

resonance. However, the value of $L s 2$ has a similar effect on both resonances. Thus, the two resonances can be flexibly controlled by jointly adjusting the dimensions of the patch and the U-shaped slot.

\section{Slotted SIW Cavities}

Once the resonances of the patch are fixed, the next step is to match the resonances of the two SIW cavities with the resonances of the patch. It is known that the resonance of a SIW cavity is determined by the size of the cavity, approximately half guided wavelengths at the resonant frequency. In this work, we find that the resonance of the cavity can also be controlled by adjusting the length of the U-shaped slot etched on the cavity. To elucidate it, the feeding networks in Fig. 1 are simplified and directly replaced by microstrip lines, as shown in Fig. 7(a). As it can be seen from Figs. 7(b) and (c), the resonant frequencies of the cavities shift to the lower band as the lengths of the slots increase, without changing the sizes of the cavities. This provides another approach to miniaturize the dimension of the SIW cavity.

\section{E. Frequency Selective Path}

Figs. 7(b) and (c) show that the antenna has a limited bandwidth and very poor isolation between the ports if the SIW cavity is fed by microstrip lines directly. In order to simultaneously increase the impedance bandwidths of the four channels and the isolation between them, frequency selective paths (FSP) based on a resonant structure are adopted in the feeds, as presented in Fig. 1. For each FSC, a pair of coupled quarter wavelengths resonators are employed to form a $2^{\text {nd }}$-order filtering circuit. The dimensions of the four groups of resonators can be approximately determined as,

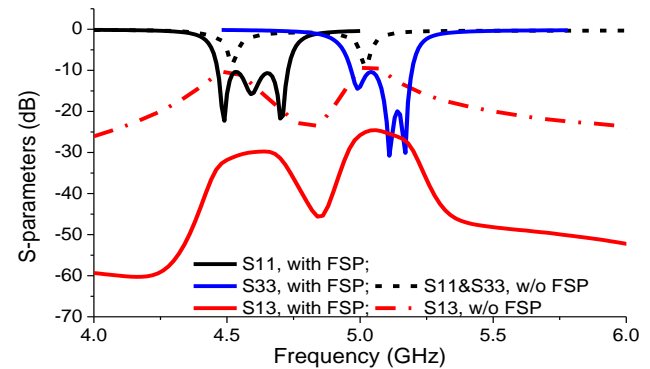

(a)

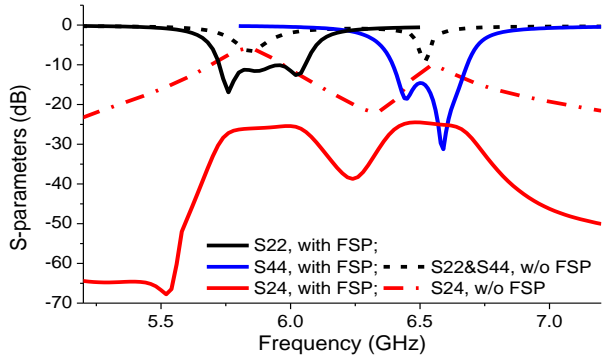

(b)

Fig. 8. Simulated S-parameters of the multiplexing filtering antenna with and without the integrated FSP, (a) lower two bands, and (b) higher two bands.

$$
L_{i 1}+L_{i 2}=L_{i 3}+L_{i 4} \approx \frac{c}{4 f_{i} \sqrt{\varepsilon_{e f f}}},(i=1,2,3,4)
$$

where, $f_{\mathrm{i}}$ is the operating frequency at the $i$-th channel, $\varepsilon_{\text {eff }}$ is the effective dielectric constant.

Fig. 8 compares the simulated S-parameters of the multiplexing filtering antenna at the two lower bands and the two higher bands with and without the frequency selective path. As observed, the port- 1 and 3 (port-2 and 4) have two identical operating bands when the patch is fed by microstrip lines directly. However, the bandwidths are very limited, and the isolation is very poor (less than $10 \mathrm{~dB}$ ). When the resonators are inserted between the SIW cavities and the microstrip lines, each port can only operate in one channel and the corresponding impedance bandwidth is significantly enhanced. In addition, the isolation between the ports can be improved from less than $10 \mathrm{~dB}$ to over $25 \mathrm{~dB}$, demonstrating the ability of the four ports to operate in the four channels independently. Thanks to the low insertion loss (less than 0.7 $\mathrm{dB}$ ) of the filtering circuit, the antenna efficiency is not obviously influenced. It is also noted that good filtering performance can be achieved for each band/channel, which is beneficial for suppressing the out-of-band interference.

\section{F. Design Guideline}

To support the design of the proposed multiplexing antenna, a step-by-step guideline is provided in the sequel. Assuming the frequencies corresponding to the four channels/ports are $f_{1}, f_{2}, f_{3}$, and $f_{4}$, respectively.

1) According to the frequencies of the four channels, two intermediate frequencies are determined, denoted as $f_{L}$ and $f_{H}$, respectively. Namely, $f_{1}<f_{L}<f_{2}, f_{3}<f_{H}<f_{4}$.

2) Design the dual-resonant slotted patch to resonate at $f_{L}$ and $f_{H}$ by using the methods presented in Part-C.

3) Design the two SIW cavities to generate two resonant frequencies which are same as the slotted patch by tuning the dimensions of the cavities and the inserted slots.

4) Assemble the two substrates and adjust the space between them to generate the four resonant frequencies required.

5) Design the four feeding networks and the corresponding frequency selective paths of the four channels, adjust the bandwidths by tuning the mutual couplings between them. 


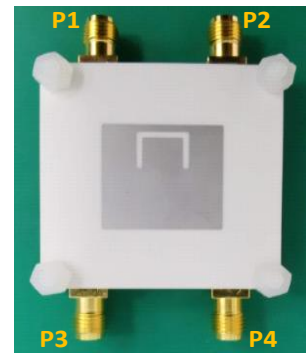

(a)

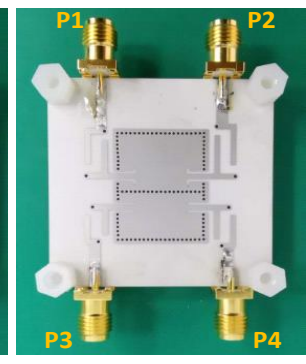

(b)
Fig. 9. Fabricated prototype of the proposed multiplexing filtering antenna: (a) top view, (b) bottom view.

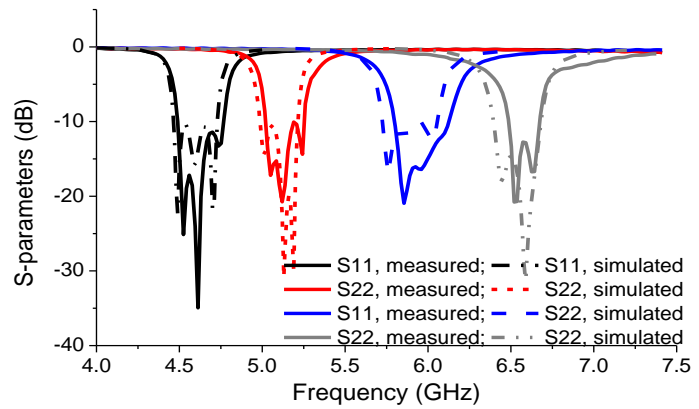

Fig. 10. Simulated and measured S-parameters of the proposed multiplexing filtering antenna.

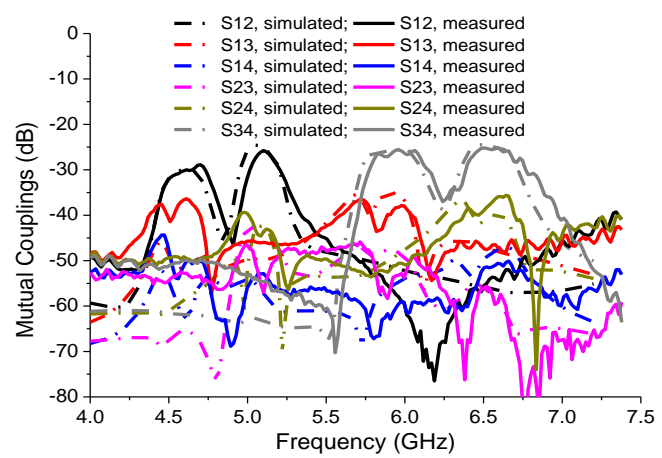

Fig. 11. Simulated and measured mutual couplings between the channels/ports of the multiplexing filtering antenna.

6) Refine each parameter and optimize the whole antenna structure to obtain the best responses in the four channels.

\section{Results AND Discussion}

Figs. 9(a)-(b) show the top and bottom views of the fabricated prototype of the proposed multiplexing filtering antenna. Four SMA connectors are used to excite the ports of the four channels/bands of a shared patch, featuring a compact size. Fig. 10 shows the simulated and measured S-parameters of the proposed multiplexing filtering antenna. As it can be observed, the antenna has four different operating bands and each of them is excited by a port as a channel. The measured results agree very well with the simulations, showing a bandwidth from 4.45 to $4.7 \mathrm{GHz}$ for channel-1 operation, a bandwidth from 4.95 to $5.2 \mathrm{GHz}$ for channel-2 operation, a bandwidth from 5.8 to $6.1 \mathrm{GHz}$ for channel-3 operation, and a bandwidth from 6.45 to $6.6 \mathrm{GHz}$ for channel-4 operation, respectively. In each band, three resonant frequencies can be observed and $3^{\text {rd }}$-order filtering responses with very good frequency selectivity are achieved. The minor discrepancy between the simulated and measured results is attributed to the fabrication and assembly tolerance.

Fig. 11 shows the simulated and measured mutual couplings between the four channels/ports of the multiplexing filtering antenna.

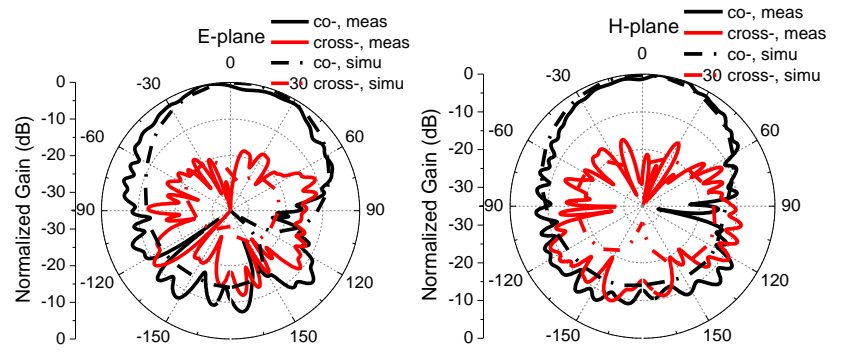

(a)
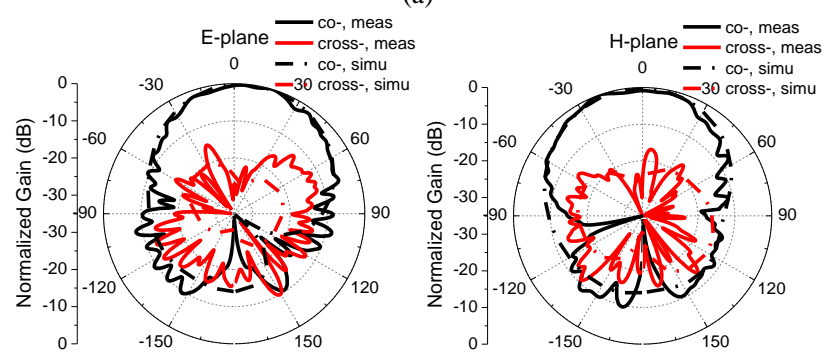

(b)

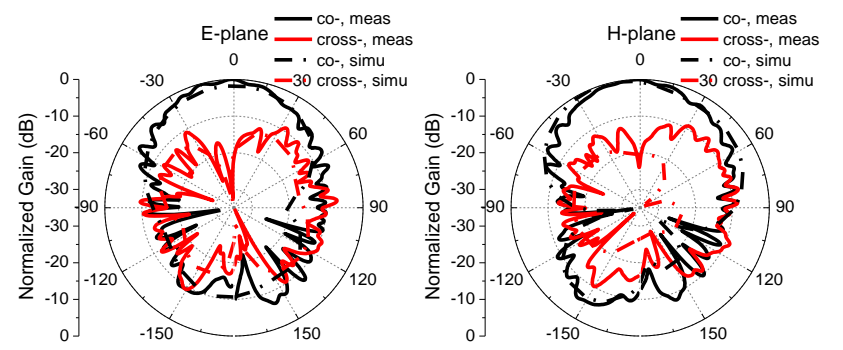

(c)
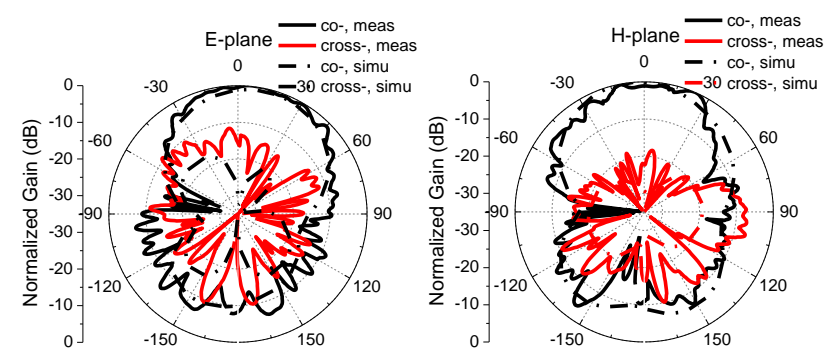

(d)

Fig. 12. Measured and simulated E- and H-plane normalized radiation patterns of the proposed multiplexing filtering antenna: (a) $4.6 \mathrm{GHz}$, port-1 is excited, (b) $5.1 \mathrm{GHz}$, port-2 is excited, (c) $6.0 \mathrm{GHz}$, port-3 is excited, (d) $6.5 \mathrm{GHz}$, port-4 is excited.

As can be observed, the antenna has a good isolation of over $25 \mathrm{~dB}$ between the four channels even though they are operating at closely spaced frequency bands and sharing the same patch as the radiating element. One can see that the main mutual coupling happens between port 1 and 2 (port 3 and 4), the isolation between port-1 and 3, port-1 and 4 , port- 2 and 3 , and port- 2 and 4 are over $37 \mathrm{~dB}$. The measured results agree very well with the simulation ones.

Fig. 12 shows the simulated and measured E- and H-plane normalized radiation patterns of the proposed multiplexing filtering antenna. The radiation patterns are measured when one port is excited and other ports are terminated with $50 \Omega$ loads. As observed, the antenna exhibits an uni-directional radiation in the broadside direction with a cross-polarization level less than $-20 \mathrm{~dB}$. The radiation patterns show that the proposed multiplexing filtering antenna has consistent radiation characteristics for the four operating channels/bands. 


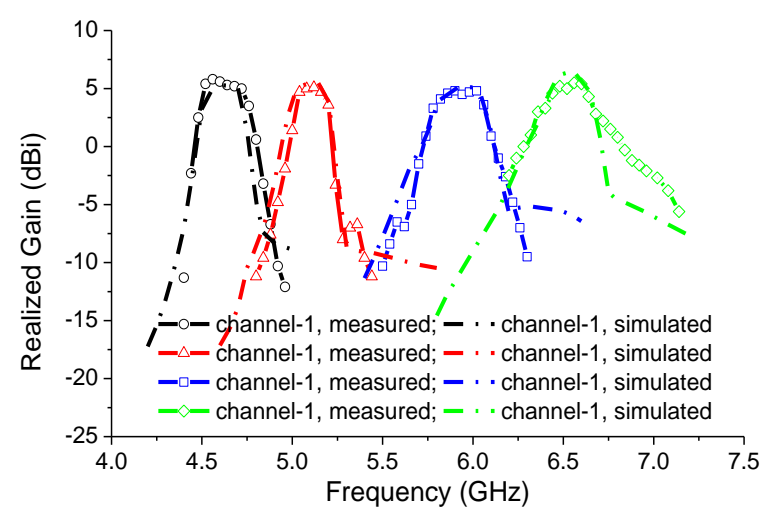

Fig. 13. Simulated and measured realized gains of the proposed multiplexing antenna when the four ports are excited, respectively.

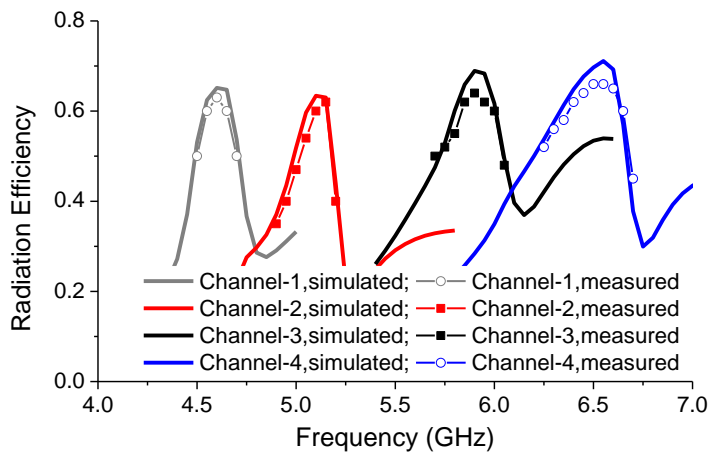

Fig. 14. Simulated and measured radiation efficiency of the proposed multiplexing antenna at four operating bands.

Fig. 13 shows the simulated and measured realized gains of the proposed multiplexing filtering antenna. The gains were measured by exciting each port and terminating the other ports, respectively. As can be seen, the antenna has a gain of about $5 \mathrm{dBi}$ for the four operating channels. For each channel, a flat gain response is achieved in the band of interest. Out of each band of interest, the gains decreased sharply by more than $10 \mathrm{~dB}$, showing a very good frequency selectivity for each channel. Fig. 14 shows the simulated and measured radiation efficiency of the proposed multiplexing antenna. Results show that the efficiency of the antenna is over $60 \%$ when it works in the four different channels. The radiation efficiency is somehow lower than traditional patch antenna, which is due to the additional cascaded frequency selective paths and the unoptimized current distribution on the patch. The measured results agree reasonably well with the simulated ones.

\section{CONCLUSIONS}

In this paper, a novel multiplexing filtering antenna with four individual ports and a shared patch has been proposed for the first time for multi-service wireless applications. The antenna has four individual ports/channels with different operating frequencies. The antenna also exhibits good filtering performance and consistent polarization in all bands. Besides, the proposed multiplexing antenna has a high isolation (over $25 \mathrm{~dB}$ ) between the four channels, making it suitable for potential multiplexing applications. The principles of achieving the four channels have been detailed with the support of a resonator-based topology and comprehensive guidelines. The antenna has been prototyped and verified experimentally, demonstrating its promising potential for multi-service/multi-channel wireless communications.

\section{REFERENCES}

[1] C. X. Mao, S. Gao, Y. Wang, F. Qin, and Q. Chu, "Multimode resonator-fed dual-polarized antenna array with enhanced bandwidth and selectivity," IEEE Trans. Antennas Propag., vol. 63, no. 12, pp. 54925499, Dec. 2015.

[2] Z. H. Jiang, D. H. Werner, "A compact, wideband circularly polarized co-designed filtering antenna and its application for wearable devices with low SAR," IEEE Trans. Antennas Propag., vol. 63, no. 9, pp. 38083818, Sep. 2015.

[3] Q. S. Wu, X. Zhang, L. Zhu, "A wideband circularly polarized patch antenna with enhanced axial ratio bandwidth via co-design of feeding network," IEEE Trans. Antennas Propag., vol. 66, no. 10, pp. 4996-5003, Oct. 2018.

[4] C. F. Ding, X. Y. Zhang, and M. Yu, "Simple dual-polarized filtering antenna with enhanced bandwidth for base station applications," IEEE Trans. Antennas Propag., early access. 2020.

[5] P. F. Hu, Y. M. Pan, X. Y. Zhang, S. Y. Zheng, "A compact filtering dielectric resonator antenna with wide bandwidth and high gain," IEEE Trans. Antennas Propag., vol. 64, no. 8, pp. 3645-3651, Aug. 2016.

[6] X. Y. Zhang, W. Duan, and Y. M. Pan, "High-gain filtering patch antenna without extra circuit," IEEE Trans. Antennas Propag., vol. 63, no. 12, pp. 5883-5888, Dec. 2015.

[7] C. X. Mao, et al., "An integrated filtering antenna array with high selectivity and harmonics suppression," IEEE Trans. Microw. Theory Techn., vol. 64, no. 6, pp. 1798-1805, Jun. 2016.

[8] P. F. Hu, Y. M. Pan, X. Y. Zhang, S. Y. Zheng, "Broadband filtering dielectric resonator antenna with wide stopband," IEEE Trans. Antennas Propag., vol. 65, no. 4, pp. 2079-2084, Apr. 2017.

[9] C. X. Mao et al., "Integrated dual-band filtering/duplexing antennas," IEEE Access, vol. 6, pp. 8403-8411, 2018.

[10] C. X. Mao, S. Gao, Y. Wang, F. Qin, Q. Chu, "Compact highly integrated planar duplex antenna for wireless communications," IEEE Trans. Microw. Theory Techn., vol. 64, no. 7, pp. 2006-2013, Jul. 2016.

[11] C. X. Mao, S. Gao, and Y. Wang, "Dual-band full-duplex Tx/Rx antennas for vehicular communications," IEEE Trans. Veh. Techn., vol. 67, no. 5, May 2018.

[12] X. J. Lin, Z. M. Xie, P. S. Zhang, and Y. Zhang, "A broadband filtering duplex patch antenna with high isolation," IEEE Antennas Wireless Propag. Lett., vol. 16, pp. 1973-1976, 2017.

[13] Y. J. Lee, J. H. Tarng, and S. J. Chung, "A filtering diplexing antenna for dual-band operation with similar radiation patterns and low cross-polarization levels," IEEE Antennas Wireless Propag. Lett., vol. 16, pp. 58-61, 2017.

[14] K. Dhwaj, X. Li, L. J. Jiang, and T. Itoh, "Low-profile diplexing filter/antenna based on common radiating cavity with quasi-elliptic response," IEEE Antennas Wireless Propag. Lett., vol. 17, pp. 1783-1787, 2018.

[15] J. F. Li, D. L. Wu, G. Zhang, Y. J. Wu, and C. X. Mao, "A left/right-handed dual circularly-polarized antenna with duplexing and filtering performance," IEEE Access, vol. 7, pp. 35431-35437, 2019.

[16] C. X. Mao, Z. H. Jiang, D. H. Werner, S. Gao, and W. Hong, "Compact self-diplexing dual-band dual-sense circularly polarized array antenna with closely spaced operating frequencies," IEEE Trans. Antennas Propag., vol. 67, no. 7, pp. 4617-4725, Jul. 2019.

[17] J. F. Li, D. L. Wu, G. Zhang, Y. J. Wu, and C. X. Mao, "Compact dual-polarized antenna for dual-band full-duplex base station applications," IEEE Access, vol. 7, pp. 72761-72769, 2019.

[18] A. A. Khan, M. K. Mandal, "Compact self-diplexing antenna using dual-mode SIW square cavity," IEEE Antennas Wireless Propag. Lett., vol. 18, pp. 343-347, 2019.

[19] P. Cheong, K. F. Chang, W. W. Choi, K. W. Tam, "A highly integrated antenna-triplexer with simultaneous three-port isolations based on multi-mode excitation," IEEE Trans. Antennas Propag., vol. 63, no. 1, pp. 363-368, Jan. 2015.

[20] A. Vosoogh, M. S. Sorkherizi, A. U. Zaman, J. Yang, and A. Kishk, "An Integrated Ka-Band Diplexer-Antenna Array module based on gap waveguide technology with simple mechanical assembly and no electrical contact requirements," IEEE Trans. Microw. Theory Techn., vol. 66, no. 2, pp. 962-972, Feb. 2018.

[21] C. X. Mao et al., "Dual-band patch antenna with filtering performance and harmonic suppression," IEEE Trans. Antennas Propag., vol. 64, no. 9, pp. 4074-4077, Sep. 2016. 